\title{
Extended Kalman Filter for Ship Tracking
}

\author{
Baoquan Suna, Bing Yan ${ }^{b}$ and JiaWei Zhang ${ }^{c}$ \\ Naval University of Engineering, Wuhan Hubei 430033, China \\ acainiaoxiaosun@163.com, byanbing_wh@yeah.net, ‘290519038@qq.com
}

Keywords: extended Kalman Filter, electric dipole, state space model.

\begin{abstract}
There are obvious electrostatic field characteristics around the ship. Eelectric dipole in three-layer medium is used to describe the characteristics of the ship. The tracking problem of electric dipole with extend Kalman Filter is studied. First, according to the movement characteristics of the target, state space model is built. Then the extend Kalman Filter is introduced. Simulation results demonstrate that this method is feasible for the field of the real-time tracking of ship with the advantage of high preciseness.
\end{abstract}

\section{Introduction}

A stable current produced in the seawater surrounding ship by the corrosion and anti-corrosion measures generates Static electric Field (SE). There are obvious distribution characteristics with SE ${ }^{[1]}$.In general, the electric field characteristics of a ship can be described by electric dipoles in an air-seawater-seabed three-layer medium. ${ }^{[2]}$

Currently, the studies on ship electric field mainly focus on the model construction and depth inversion ${ }^{[3]}$. There are few reports on the application of ship electric field in the tracking and positioning of ships. Our present study aims to track target ship through ship SE.

The Kalman filter (KF) was first proposed by R. E. Kalman and it is a linear minimum variance estimate. The extended Kalman filter (EKF) is designed to suit for non-linear systems. Compared to other filtering methods, EKF calculates a small amount with a high accuracy. So, the method of this paper is based on EKF.

\section{Ship SE Tracking Model}

\subsection{State Space Model}

The general form of the state space model is ${ }^{[4]]}$

$\boldsymbol{x}_{k}=\boldsymbol{a}\left(\boldsymbol{x}_{k-1}\right)+\boldsymbol{w}_{k-1}$

$\boldsymbol{y}_{k}=\boldsymbol{h}\left(\boldsymbol{y}_{k-1}\right)+\boldsymbol{v}_{k}$

$\boldsymbol{x}_{k} \in \mathbb{R}^{n}$ is the state vector in the dimension of $\mathrm{n}$ at the moment $\mathrm{k}$; $a$ is the state transfer function; $h$ is the observation function; $\boldsymbol{w}_{k}$ is the state noise vector, $\boldsymbol{v}_{k}$ is the observation noise vector, $\boldsymbol{w}_{k-1} \sim \mathcal{N}\left(\boldsymbol{\theta}, \boldsymbol{Q}_{k-1}\right), \boldsymbol{v}_{k} \sim \mathcal{N}\left(\boldsymbol{\theta}, \boldsymbol{R}_{k}\right)$ and $\boldsymbol{Q}_{k-1}$ is the covariance matrix for process noise, and $\boldsymbol{R}_{k}$ is the covariance matrix for observing noise. $\left\{\boldsymbol{w}_{k-1}\right\}$ And $\left\{\boldsymbol{v}_{k}\right\}$ are independent of each other.

The ship SE model can be described by level electric dipoles in an air-seawater-seabed three-layer medium. In the sensor coordinate system, for one electric dipole $I=\left[I_{x}, I_{y}\right]$.As shown in Figure 1 . Then ${ }^{[5]}$ 


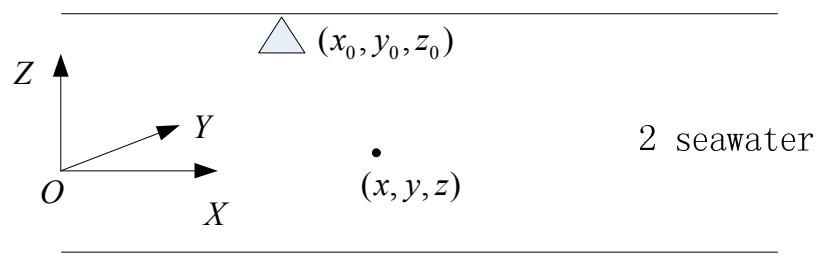

3 seabed

Figure 1. Air - seawater - seabed three - layer uniform

Media coordinate system

$$
\begin{aligned}
& \Phi(x, y, z)=\Phi_{x}(x, y, z)+\Phi_{y}(x, y, z) \\
& =\sum_{k=1}^{\infty}\left[\begin{array}{c}
\frac{\eta^{k} I_{x} \mathrm{~d} l\left(x-x_{0}\right)}{4 \pi \sigma_{1} r_{1 k}{ }^{3}}+\frac{\eta^{k} I_{y} \mathrm{~d} l\left(y-y_{0}\right)}{4 \pi \sigma_{1} r_{1 k}{ }^{3}} \\
+\frac{\eta^{k} I_{x} \mathrm{~d} l\left(x-x_{0}\right)}{4 \pi \sigma_{1} r_{2 k}{ }^{3}}+\frac{\eta^{k} I_{y} \mathrm{~d} l\left(y-y_{0}\right)}{4 \pi \sigma_{1} r_{2 k}{ }^{3}}
\end{array}\right]+\sum_{m=0}^{\infty}\left[\begin{array}{c}
\frac{\eta^{m} I_{x} \mathrm{~d} l\left(x-x_{0}\right)}{4 \pi \sigma_{1} r_{1 m}{ }^{3}}+\frac{\eta^{m} I_{y} \mathrm{~d} l\left(y-y_{0}\right)}{4 \pi \sigma_{1} r_{1 m}{ }^{3}} \\
+\frac{\eta^{m} I_{x} \mathrm{~d} l\left(x-x_{0}\right)}{4 \pi \sigma_{1} r_{2 m}{ }^{3}}+\frac{\eta^{m} I_{y} \mathrm{~d} l\left(y-y_{0}\right)}{4 \pi \sigma_{1} r_{2 m}{ }^{3}}
\end{array}\right]
\end{aligned}
$$

Where $\left(x_{0}, y_{0}, z_{0}\right)$ is the location of electric dipole, $(x, y, z)$ is a point in the sea, $\sigma$ is the seawater conductivity, $\sigma_{1}$ is the seabed conductivity, $\eta=\left(\sigma_{1}-\sigma_{2}\right) /\left(\sigma_{1}+\sigma_{2}\right)$ is the submarine reflection coefficient, $\vec{r}_{1 k}, \vec{r}_{2 k}, \vec{r}_{1 m}, \vec{r}_{2 m}$ are respectively

$$
\begin{aligned}
& \vec{r}_{1 k}=\left(x-x_{0}\right) \vec{i}+\left(y-y_{0}\right) \vec{j}+\left(z-2 k D+z_{0}-2 h\right) \vec{k} ; \\
& \vec{r}_{2 k}=\left(x-x_{0}\right) \vec{i}+\left(y-y_{0}\right) \vec{j}+\left(z-2 k D-z_{0}\right) \vec{k} ; \\
& \vec{r}_{1 m}=\left(x-x_{0}\right) \vec{i}+\left(y-y_{0}\right) \vec{j}+\left(z+2 m D-z_{0}\right) \vec{k} ; \\
& \vec{r}_{2 m}=\left(x-x_{0}\right) \vec{i}+\left(y-y_{0}\right) \vec{j}+\left(z+2 m D+z_{0}-2 h\right) \vec{k} .
\end{aligned}
$$

$D$ is the depth of seawater.

The three-component electrostatic field is the negative derivative of the potential in three directions, respectively:

$$
\begin{aligned}
& E_{x}=-\frac{\partial \Phi(x, y, z)}{\partial x} \\
& E_{y}=-\frac{\partial \Phi(x, y, z)}{\partial y} \\
& E_{z}=-\frac{\partial \Phi(x, y, z)}{\partial z}
\end{aligned}
$$

The target signal measured at the No. $j$ triaxial electric field sensor can be modeled as follows:

$\boldsymbol{y}^{(j)}\left(\boldsymbol{x}_{k}\right)=\boldsymbol{h}^{(j)}\left(\boldsymbol{x}_{k}\right)+n_{k}^{(j)}=\boldsymbol{E}_{k}^{(j)}+n_{k}^{(j)}$

Where $\boldsymbol{E}_{k}^{(j)}=\left[\boldsymbol{E}_{x, k}^{(j)}, \boldsymbol{E}_{y, k}^{(j)}, \boldsymbol{E}_{z, k}^{(j)}\right]$ and $n_{k}^{(j)}=\left[n_{x, k}^{(j)}, n_{y, k}^{(j)}, n_{z, k}^{(j)}\right]$ are the measured signals and noises, respectively.

Further observations of multiple sensors are available

$\boldsymbol{y}_{k}=\left[\begin{array}{c}h^{(1)}\left(\boldsymbol{x}_{k}\right) \\ h^{(2)}\left(\boldsymbol{x}_{k}\right) \\ \vdots \\ h^{(J)}\left(\boldsymbol{x}_{k}\right)\end{array}\right]+\left[\begin{array}{c}n_{k}^{(1)} \\ n_{k}^{(2)} \\ \vdots \\ n_{k}^{(J)}\end{array}\right]=\boldsymbol{h}\left(\boldsymbol{x}_{k}\right)+\boldsymbol{n}_{k}=\boldsymbol{h}\left(\boldsymbol{x}_{k}\right)+\boldsymbol{v}_{k}$

The state of the ship target contains the target position, speed, and point currents line array $\boldsymbol{x}_{k}=\left[\boldsymbol{r}_{k}, \boldsymbol{v}_{k}, I_{p}^{1: N}\right]^{\mathrm{T}}$

Where $\boldsymbol{r}_{k}=[x, y, z]^{\mathrm{T}}, \boldsymbol{v}_{k}=\left[v_{x}, v_{y}\right]^{\mathrm{T}}, I_{k}^{1: N}=\left[I_{1}, I_{2}, \ldots, I_{N}\right]^{\mathrm{T}}$. 
The ship target is characterized by low dynamic characteristics and its motion state can be simply modeled as DWNA (Discrete White Noise Acceleration) model. The motion equation is

$\mathcal{X}_{k}=\boldsymbol{\Phi}_{m} \mathcal{X}_{k-1}+\boldsymbol{\Gamma}_{m} \mathcal{W}_{k-1}$

Where $\boldsymbol{\Phi}_{m}=\left[\begin{array}{cc}\boldsymbol{I}_{3 \times 3} & T_{s} \boldsymbol{I}_{2 \times 2} \\ \boldsymbol{0}_{2 \times 3} & \boldsymbol{I}_{2 \times 2}\end{array}\right]$ is motion state transition matrix, $\boldsymbol{\Gamma}_{m}=\left[\begin{array}{c}\frac{T_{s}^{2}}{2} \boldsymbol{I}_{2 \times 2} \\ \boldsymbol{0}_{2 \times 1} \\ T_{s} \boldsymbol{I}_{2 \times 2}\end{array}\right]$ is noise gain matrix, and $T_{s}$ is sampling time interval.

The DWNA model assumes that the acceleration is Gaussian white noise, i.e.

$\boldsymbol{\Sigma}=E\left[\boldsymbol{w}_{k} \boldsymbol{w}_{k}^{\mathrm{T}}\right]=\operatorname{diag}\left(\sigma_{x}^{2}, \sigma_{y}^{2}\right)$

Electric dipole is a constant, with a relatively small noise. The whole state equation is

$\boldsymbol{x}_{k}=\boldsymbol{F} \boldsymbol{x}_{k-1}+\boldsymbol{w}_{k-1}$

Where $\boldsymbol{F}=\left[\begin{array}{cc}\boldsymbol{\Phi}_{m} & \boldsymbol{0}_{5 \times N} \\ \boldsymbol{0}_{5 \times N} & \boldsymbol{I}_{N \times N}\end{array}\right]$, the noise covariance matrix is $\boldsymbol{Q}=\left[\begin{array}{c}\boldsymbol{\Gamma}_{m} \boldsymbol{\Sigma} \boldsymbol{\Gamma}_{m} \\ \alpha \boldsymbol{I}_{N \times 2}\end{array}\right]$, and $\alpha$ is a small constant.

\subsection{Extended Kalman Filter}

The first-order Taylor expansion of the observation equation is carried out to obtain a filtering algorithm that can handle the nonlinear observation system, and this is EKF ${ }^{[6]}$

$\boldsymbol{y}_{k}=\boldsymbol{h}\left(\boldsymbol{x}_{k}\right)+\boldsymbol{v}_{k}=\boldsymbol{h}\left(\hat{\boldsymbol{x}}_{k \mid k-1}\right)+\hat{\boldsymbol{H}}_{k}\left(\boldsymbol{x}-\hat{\boldsymbol{x}}_{k \mid k-1}\right)+\boldsymbol{v}_{k}$

$\hat{\boldsymbol{H}}_{k}=\left.\frac{\partial \boldsymbol{h}}{\partial \boldsymbol{x}}\right|_{\boldsymbol{x}=\hat{\boldsymbol{x}}_{k k-1}}$

$\hat{\boldsymbol{x}}_{k \mid k-1}$ is the linearization of the expansion point and $\hat{\boldsymbol{H}}_{k}$ is the Jacobian matrix of $\boldsymbol{h}\left(\boldsymbol{x}_{k}\right)$ at $\hat{\boldsymbol{x}}_{k \mid k-1}$.

\section{Simulation Results and Analysis}

The boat sailed on the water, so we ignore the motion in z-axis. Define the root mean square error varying with the number of iterations of the filter as follows

$R M S E_{k}=\sqrt{\frac{1}{M C} \sum_{i=1}^{M C} \operatorname{tr}\left[\left(\boldsymbol{r}_{k}-\hat{\boldsymbol{r}}_{k}\right)\left(\boldsymbol{r}_{k}-\hat{\boldsymbol{r}}_{k}\right)^{T}\right]}$

Where $M C$ is the number of simulations

Consider the simulation scenario in Table 1-2.

Table 1. Simulation scene parameters

\begin{tabular}{|c|c|c|}
\hline \multicolumn{2}{|c|}{ Parameter(unit) } & Magnitude \\
\hline \multicolumn{2}{|c|}{$\boldsymbol{r}_{0} /(\mathrm{m})$} & {$[-80,80,52]$} \\
\hline \multicolumn{2}{|c|}{$\boldsymbol{v}_{0} /(\mathrm{m} / \mathrm{s})$} & {$[10,10]$} \\
\hline \multicolumn{2}{|c|}{$\boldsymbol{I}_{0} /(\mathrm{A})$} & 150 \\
\hline \multicolumn{3}{|c|}{ Table 2. Filter initial condition } \\
\hline Parameter(unit) & Initial value & Initial mean square error \\
\hline$\hat{\boldsymbol{r}}_{0} /(\mathrm{m})$ & {$[-160,160,52.1]$} & $\operatorname{diag}\left[80^{2}, 80^{2}, 0.01\right]$ \\
\hline$\hat{\boldsymbol{v}}_{0} /(\mathrm{m} / \mathrm{s})$ & {$[12,12]$} & $\operatorname{diag}\left[2^{2}, 2^{2}\right]$ \\
\hline$\hat{\boldsymbol{I}}_{p} /(\mathrm{A})$ & 140 & 100 \\
\hline
\end{tabular}

The results are shown in Figure 2-3 


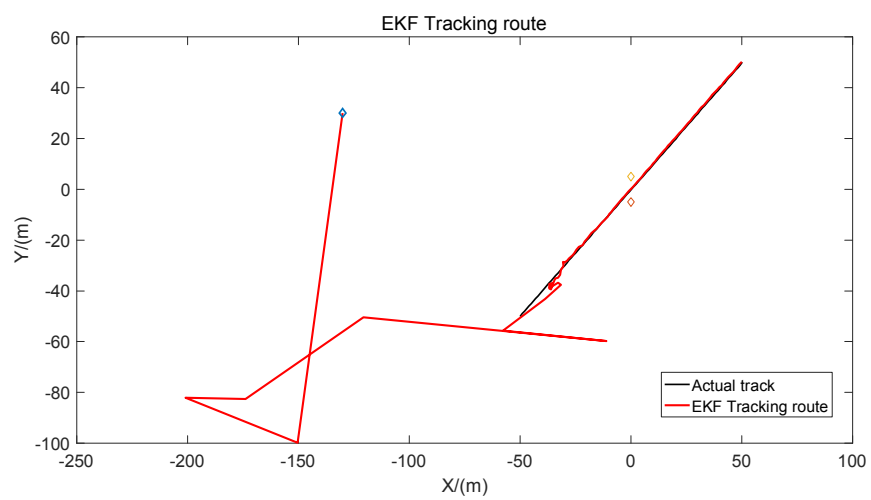

Figure 2. EKF tracking course diagram

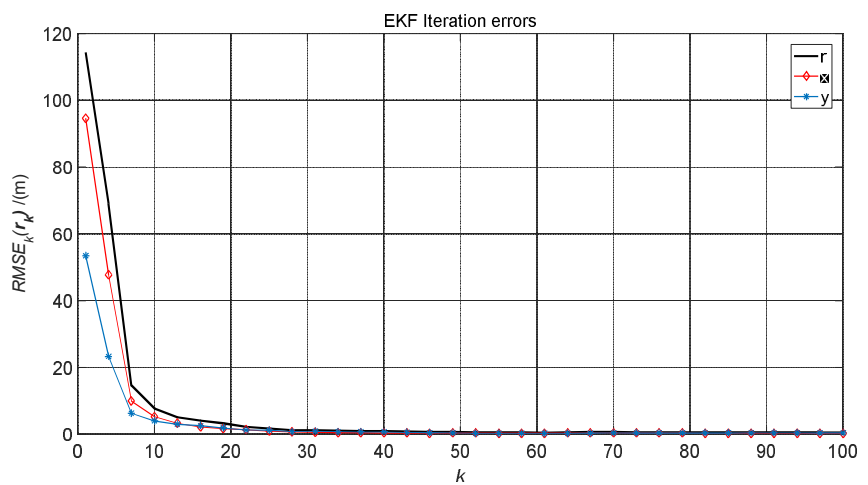

Figure 3. The iteration errors with the number of iterations

Figure 1 shows the tracking course. The red line is the tracking course using EKF. The black line is the actual route. And we can see, although the initial error is huge, the target could be tracked quickly and effectively. Figure 2 shows the iteration errors with the number of iterations. The black line represents the $r\left(\sqrt{\left(x^{2}+y^{2}\right)}\right)$ error, the red line represents the $x$ error, and the blue line represents the $y$ error. We can see that after a period of iterations, the error decreasing rapidly. Near the sensor the errors are approximately equal to 0 .

\section{Conclusion}

In this study, we try to track and locate ship targets by ship SE signal. The simulation results demonstrate that the EKF algorithm can effectively track and locate the ship with high accuracy, which is of great significance in the application of ship SE signal.

\section{Acknowledgments}

This study is sponsored by National Natural Science Foundation of China Youth Project (No.51509252).

\section{References}

[1]. Chen Cong. Research on ship electromagnetic field model and depth conversion [D]. Wu Han: the Naval University of Engineering, 2008. (in Chinese)

[2]. Cong Chen, Shenguang Gong, Dingguo Li. Research on the Static Magnetic Field Related with Corrosion and Anticorrosion of Ship Based on the Electric Dipole Model [J]. ACTA ARMAMENTARII, 2010, 31(1): 113-118. (in Chinese)

[3]. Cong Chen, Zhiguo Jiang, Lufeng Yao, et al. Characteristic analysis of corrosion-related static electromagnetic field produced by a submarine in shallow sea[J]. Journal of Nava University of Engineering, 2014(3):1-6.(in Chinese) 
[4]. Guibert A, Chadebec O, Coulomb J L, et al. Ship's Hull Corrosion Diagnosis From Close Measurements of Electric Potential in the Water[J]. IEEE Transactions on Magnetics, 2009, 52(3):1828-1831.

[5]. Adey R, Baynham J M W, Lodge A. Predicting Corrosion Related Signatures [J]. Simulation of Electrochemical Processes II, 2007.

[6]. Simon D. Optimal State Estimation [M]. New York: John Wiley\&Sons, 2006.

[7]. Cong Chen, Dingguo Li, Zhiguo Jiang, et al. Electric field of a static electric dipole in three-layer-medium model using secondary equivalent method[J]. Acta Phys. Sin. 2012, 61(24): 1-7. (in Chinese)

[8]. Jazwinski A. Stochastic process and filtering theory. New York: Academic Press, 1970. 